\section{Basic rest-activity cycle}

Helga Peter ${ }^{1}$ und Thomas Penzel ${ }^{2}$

${ }^{1}$ Marburg, Deutschland

${ }^{2}$ Interdisziplinäres Schlafmedizinisches Zentrum, Charité Universitätsmedizin Berlin, Berlin, Deutschland

\section{Synonyme}

BRAC; Ruhe-Aktivitäts-Zyklus

\section{Definition}

Beim Menschen sind über die 90-minütigen NREM-REMZyklen im Nachtschlaf hinaus eine Reihe weiterer ultradianer Rhythmen bekannt, die von ihrer Dauer her ebenfalls im Bereich von etwa 90 Minuten liegen. Dazu zählen beispielsweise der Rhythmus der gastrointestinalen Spontanmotorik (siehe "Gastrointestinale Motilität") und die spontanen Veränderungen des Pupillendurchmessers. Letztere signalisieren die Spontanschwankungen der Daueraufmerksamkeitsleistung und sie können mittels Pupillometrie objektiviert werden ( „Pupillographischer Schläfrigkeitstest“). 